\title{
JCWAEED: JOINT CHANNEL ASSIGNMENT AND WEIGHTED AVERAGE EXPECTED END-TO-END DELAY ROUTING PROTOCOL IN WIRELESS MESH NETWORKS
}

\author{
Wang Yi-rong ${ }^{1}$, Wang Yan-ru ${ }^{2}$, Zhang $\mathrm{Hao}^{3}$, Liu Kai-ming and Li Nan \\ ${ }^{1,2}$ Beijing Guodiantong Network Technology Co.Ltd., Beijing, China \\ ${ }^{3}$ State Grid Shandong Electric Power Corporation, \\ Economic and Technical Research Institute, Shandong, China
}

\begin{abstract}
In recent years, multi-channel multi-radio Wireless Mesh network has become one of the most important technologies in the evolution of next-generation networks. Its multi-hop, selforganization, self-healing and simple deployment is an effective way to solve the bottleneck problem of last mile. In this paper, we propose a new routing metric called WAEED, deployed in JCWAEED protocol, a joint channel assignment and weighted average expected end-to-end delay routing protocol which considers both interference suppression with factor IF and end-toend delay. Additionally, we give the exact calculation formula of transmission delay and queuing delay. Simulations results demonstrate that JCWAEED outperforms other joint design routing protocols in terms of throughput, end-to-end delay and packet loss rate.
\end{abstract}

\section{KEYWORDS}

Multi-channel Multi-radio, Wireless Mesh Networks, End-to-end Delay Control, Joint Design, Channel Assignment, Routing Protocols

\section{INTRODUCTION}

Multi-radio multi-channel Wireless Mesh Network is one of the most important technologies in the evolution of next-generation networks. Its multi-hop, self-organization, self-healing and simple deployment is an effective way to solve the bottleneck problem of last mile. However, with the increase of the node density, it will cause the interference situation to deterioration, the channel utilization rate is low and other rigid environment. Considering the interaction between channel allocation and routing, it needs joint channel allocation and routing to optimize the design to improve the channel utilization and network performance, and play the advantages of multiradio multi-channel technology. Since it is a challenging interdependent task to find routing paths and allocate channels to links in the WMN, its focus is joint schemes about channel assignment and routing in multi-channel multi-radio wireless mesh networks.

For the moment, there are two groups for joint channel assignment and routing schemes in multichannel multi-radio wireless mesh networks [1]. One is called sequential channel assignment and 
routing protocol design [2-5], and the strategy is that designers treat channel assignment and routing as two independent processes. People usually do channel assignment optimization and routing optimization separately in sequence. Nevertheless, we cannot acquire network request for communications information before channel assignment in reality. The other one is joint channel assignment and routing protocol design [6-8], and people treat channel assignment as a whole procedure for optimization. Specifically, this NP-hard problem can be turned into liner programming with some certain objective by setting constraint conditions for joint optimization. This strategy solution, nonetheless, is of both very high algorithm complexity and extraordinarily high time complexity .In addition, the approximate solutions are generally lack of strict theoretical support [9].

The paper further analyzes and compares the research status of joint optimal design of channel allocation and routing in multi-radio multi-channel wireless Mesh networks. In this paper, the necessity and feasibility of joint optimal design of channel allocation and routing in multi-radio multi-channel wireless Mesh networks are expounded in detail by referring to a lot of literatures.

Based on the network model and protocol interference model, the mathematical model is carried out and the calculation method of average end-to-end delay is deduced out of a lot of theoretical research and simulation analysis. The HELLO packet is designed as the detection packet of network information, used to obtain the relevant network parameters in the network, and finally introduce the interference penalty factor IF, used to characterize the node using the interference of channel i. In this paper, the appropriate channel is selected by the size of the IF, and the routing metric is designed in combination with the average end-to-end delay. Based on the above work, this paper proposes an efficient joint channel allocation and routing optimization method (JCWAEED), considering delay control, interference suppression, channel allocation and routing establishment. The JCWAEED algorithm utilizes different channel combinations to obtain the set of single-hop routing metric WAEED from the node to the next hop based on the single hop metric. And the channel with the minimum single-hop routing metric in the set is taken as the best channel for the link from the node to the next hop. The performance of JCWAEED algorithm, including the number of streams and the rate of flow, is analyzed and compared with that of the existing protocols.

The paper is organized as follows. Section 1 introduce basic concepts and infrastructure of multiradio multi-channel wireless Mesh networks, and describe the design idea and key technologies of multi-radio multi-channel Mesh networks in detail. Section 2 gives a exact description of some relative works about joint design of channel assignment and routing protocols. Section 3 demonstrates system model used in this paper. Section 4 proposes joint channel assignment and weighted average expected end-to-end delay routing protocol. Section 5 is our simulation results, and gives analysis of our protocol advantages in terms of network throughput, end-to-end delay, packet loss and channel utilization rate. Section 6 gives final conclusion of this paper.

Our major contributions can be summarized as follows.

1. We give average end-to-end delay in exact and strict theoretical calculation including transmission delay and queuing delay.

2. We provide more efficient way of interference factor IF between channels calculation during channel assignment period.

3. Considering two points above, we propose a new routing metric WAEED, deployed in JCWAEED protocol to conduct routing in MRMC-WMN. 


\section{RELATIVE WORKS}

Channel assignment and routing design depend on each other can remedy weakness of each other .Hence the joint design can significantly improve network performance.

The first group of optimization is to treat channel assignment and routing as two independent processes optimizing them in sequence, and then select the appropriate channel according to the flow distribution in the network [10].

JCEED algorithm [2] assign channels after routing, considering end-to-end delay and queuing delay as routing metric and adjust channels allocation along with routing recursively with feedback information of EED until making total network interference deduction to minimum. Within every recursion, choose the path with the minimum delay, and choose the optimum channel according to the channel assignment algorithm. It decreases interference between channels by joint routing and channel assignment optimization under the limits of time delay. However, it does not give an exact and accurate computing method about end-to-end delay derivation. It is not comprehensive only using numbers of successfully received packets under channel $\mathrm{i}$ as index to represent the interference. Channel assignment initialization is not thorough enough, if co-channel were randomly assigned to different radios of one node, it will not only increase the channel switching times, but also increase the interference of other nodes assigned to the channel. Channel allocation process can be further optimized by reducing final establishing routing time.

The second group is joint channel assignment and routing protocol design [6][7][8], and people treat channel assignment as a whole procedure for optimization. Specifically, this NP-hard problem can be turned into liner programming with some certain objective by setting constraint conditions for joint optimization. This strategy solution, nonetheless, is of both very high algorithm complexity and extraordinarily high time complexity.

The key of channel allocation and routing joint optimization design is: network connectivity, network traffic and link-state information, technical limitations and the design of the routing metric [6]. Plus, routing metric design considers some key factors: the path hop, channel interference, radio interface switch [7], computational complexity decrement, convergence time decrement and local constraints [8].

\section{SYSTEM MODEL}

\subsection{Network model}

We focus on the study of the multi-radio multi-channel wireless Mesh network, and MRMC WMN network topology can be expressed as a directed graph $G(N, L), N$ denotes the set of network nodes, $L$ denotes set of physical wireless links. $C_{k}=\{1,2,3\}$ denotes available channels set of multi-channel multi-radio wireless Mesh network. Wireless link $l_{u v}$ denotes the assigned radios from the sending node $u$ to the receiving node $v$, assigning available channel $\mathrm{k}$ for the link can be expressed as $l_{u v}^{k}$. In order to facilitate comprehension, the table below gives the key symbols meaning used in the paper. 
Table 1. Key symbols in MRMC WMN

\begin{tabular}{|l|l|l|l|}
\hline$C_{k}$ & Available channels set & $R_{t}$ & Node transmission range \\
\hline $\mathrm{L}$ & Physical wireless links set & $R_{u}$ & $\begin{array}{l}\text { Radio number configured for the } \\
\text { node } \mathrm{u}\end{array}$ \\
\hline $\mathrm{G}$ & Network directed graph & $C(u)$ & $\begin{array}{l}\text { Assigning available channels for } \\
\text { node u }\end{array}$ \\
\hline$l_{u v}^{k}$ & $\begin{array}{l}\text { available channel k for the link } \\
\text { between node u and node } \mathrm{v}\end{array}$ & $\mathrm{N}$ & mesh nodes set \\
\hline$R_{s}$ & Node carrier sense range & $R_{i}$ & node interference range \\
\hline
\end{tabular}

Because the number of radio for each wireless Mesh node configuration is limited, assigning channel number for the wireless Mesh nodes does not exceed the number of available radios wireless Mesh nodes, at the same time in order to improve the utilization rate of spectrum, different radios should be assigned with different channels, which means all of the radios of a wireless mesh node will not be assigned with the same channel. In addition, theoretically the radios of node interface can't be more than available orthogonal channels, there is a relationship as follows:

$$
|C(u)| \leq R_{u} \leq k, C(u) \subseteq C_{k}
$$

\subsection{Interference model}

This paper adopts the Protocol interference Model which is commonly used. In MRMC - WMN, effective communication range of the wireless Mesh network nodes can be classified into three parts: node Transmission Range $R_{t}$, node Carrier Sensing Range $R_{s}$ and node Interference Range $R_{t}$. Transmission range $R_{t}$ denotes maximum range of mesh node successfully receiving signal without any interference, in general, the mesh node's transmission range is related to its transmission power, frequency. And node transmission power is proportional to the node transmission range; Carrier sensing range $R_{s}$ represents maximum range of nodes detecting other carrier signal sent by other nodes; node interference range $R_{i}$ denotes conflict range caused by nodes interference .In general, relationship is as follows:

$$
R_{t}<R_{i}=1.78 R_{t}<R_{s}=2.2 R_{t}
$$

When there is transmission requirement, link $\mathrm{a}-\mathrm{b}$ and link $\mathrm{f}-\mathrm{g}$, since the mesh node $\mathrm{b}$ is within interference range of link $f-g$, but not within the communication range of node $f$, when link $a-b$ and link f-g are assigned with the same channel or orthogonal channel.

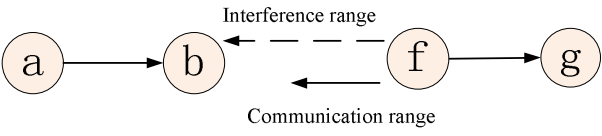

Figure 1. Relationship between interference and communication range

As shown, interference in WMNs is classified into two types: intra-flow and inter-flow interference. If different simultaneous transmissions of the same data flow interfere with each other, we call it intra flow interference. On the other hand, for simultaneous transmissions of different data flows, inter- flow interference instead. If the mutual interference of link can be assigned with different channels, then the two kind of interference can be reduced appropriately. 


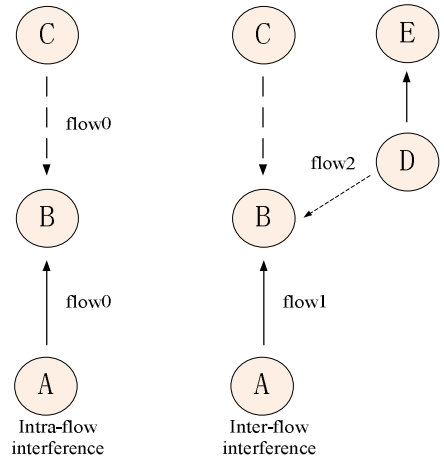

Figure 2. Intra-flow interference and inter-flow interference

\section{JOINT CHANNEL ASSIGNMENT AND WEIGHTED AVERAGE EXPECTED END-TO-END DELAY ROUTING PROTOCOL}

In the part, we will introduce a joint channel assignment and delay routing protocol with weight average expected end-to-end delay.

$$
W A E E D_{n, m}^{c}=(1-\beta) E E D_{i}+\beta I F_{i}
$$

$W A E E D_{n, m}^{c}$ denotes weighted average expected end-to-end delay from node $\mathrm{n}$ to node $\mathrm{m}$ by channel c, where $E E D_{i}$ denotes average end-to-end delay by channel i. As to $I F_{i}$ denotes interference by channel $\mathrm{i}$, plus $I F_{i} \geq 1$, that $I F_{i}$ is closer to 1 means interference by channel i is smaller. $M_{n, i}$ denotes packets from node $\mathrm{n}$ to node $\mathrm{m}$ in total. $\sum p k_{m, i}$ denotes packets successfully received by node $\mathrm{m}$. $\beta$ is a weighted factor, $0 \leq \beta \leq 1$, usually $\beta$ is set as 0.5 .

JCWAEED algorithm adopts contrast of the IF interference and the available channel end-to-end delay EED to choose the most appropriate channels, and in the process of channel allocation we need to follow these two principles: (1) for different radio of one node should be assigned with different orthogonal channels. (2) one radio unbounded with channel should be assigned appropriate channel in priority. While assigning channels, calculate routing metrics WAEED, then get the routing metrics collection, and select the result of channel allocation and routing with the minimum routing metric value .

\subsection{Expected End-to-end delay}

The end-to-end delay of a routing path is the sum of total hops delay along this path, including transmission delay along the links and queuing delay. For convenience, we define EED to represent the average end-to-end delay on the link.

To calculate routing metrics EED with the available channel $\mathrm{i}$ on the wireless link, each node needs to monitor the number of cache packages over the network layer waiting for MAC layer service, as well as the measure MAC layer transmission failure probability, the measurement of packet loss rate. Transmission failure may be caused by the MAC layer interference or the poor quality of channel. Link i, therefore, the end-to-end delay on the average represented as:

$$
E E D_{i}=E\left[T_{i}+Y_{\mathrm{i}}\right]
$$


$T_{i}$ denotes transmission delay over the link i, $Y_{i}$ denotes queuing delay, $E E D_{i}$ the average delay of a data packet over the link i. Assume that a new packet on the link i has entered a packet buffer queue, we define the average end-to-end delay as:

$$
E E D_{i}=\left(Q_{i}+1\right) E\left[T_{i}\right]
$$

$E E D_{i}$ consists of transmission delay and queuing delay. For $E E D_{i}$ calculation, as long as we derive the value of $E\left[T_{i}\right]$, then we can get the value $E E D_{i}$.In particular, for CSMA protocol, transmission delay includes not only backofftime delay but also channel busy time due to the other node transmission. We define packet loss rate over the link $\mathrm{i}$ as $p_{i}$, define the service time as $T_{i}$, define maximum retransmission times as $\mathrm{K}, W_{j}$ denotes the jth backoff stage competition window, $\mathrm{L}$ denotes packet size ,B denotes link bandwidth. If we ignore the constraints of backoff period, then $E\left[W_{j}\right]=\left(w_{j}-1\right) / 2$. Therefore, average transmission delay $E\left[T_{i}\right]$ can be represented as follows:

$$
E\left[T_{i}\right]=E[\text { transmissiontime }]+E[\text { backofftime }]
$$

Plus,

$$
\begin{aligned}
\text { E[backofftime }] & =\sum_{k=1}^{K+1} p_{i}^{k-1}\left(1-p_{i}\right)^{I\{k<K+1\}} \sum_{j=1}^{k} E\left[W_{j}\right] \\
& =\frac{W_{\min }\left[1-\left(2 p_{i}\right)^{K+1}\right]}{2\left(1-2 p_{i}\right)}-\frac{1-p_{i}{ }^{K}}{2\left(1-2 p_{i}\right)}
\end{aligned}
$$

Hence, average transmission delay $E\left[T_{i}\right]$ can be denoted as follows.

$$
\begin{aligned}
E\left[T_{i}\right] & =E[\text { transmissiontime }]+E[\text { backofftime }] \\
& =\frac{L}{B} \sum_{k=1}^{\infty} k \cdot p_{i}^{k-1} \cdot\left(1-p_{i}\right)+\sum_{k=1}^{\infty} p_{i}^{k-1}\left(1-p_{i}\right) \sum_{j=1}^{k} E\left[W_{j}\right] \\
& =\frac{L}{B\left(1-p_{i}\right)}+\frac{W_{\min }}{2\left(1-2 p_{i}\right)}-\frac{1}{2\left(1-p_{i}\right)}
\end{aligned}
$$

Multi-radio multi-channel wireless Mesh network has the characteristics of multi-hops network, considering there might be $\mathrm{H}$ hops along the end-to-end path, so the average end-to-end delay EED can be defined as:

$$
E E D=\sum_{i=1}^{H} E E D_{i}
$$

\subsection{Interference calculation}

We define channel interference factor as IF, which can represent interference between cochannels and different channels, therefore, for the channel i, its channel interference can be defined as:

$$
I F_{i}=\frac{M_{n, i}}{\sum p k_{m, i}}
$$

$I F_{i}$ denotes interference using channel $\mathrm{i}$, and $I F_{i} \geq 1, I F_{i}$ the closer to 1 indicates the smaller interference using channel $\mathrm{i}$, which can be negligible. $M_{n, i}$ denotes the total number of packages which node $\mathrm{n}$ sends to node $\mathrm{m}$. $\sum p k_{m, i}$ denotes the total number of packets node m successfully 
received from node $\mathrm{n}$. We define the IF by proportional relations, which can represent interference more accurately, thus select more appropriate channel to reduce the co-channel interference.

\subsection{Routing metric}

From part 4.1 and 4.2 above, we can conclude the formula of the average end-to-end delay EED and channel interference factor IF, joint routing metric is expressed as:

$$
W A E E D_{n, m}^{c}=(1-\beta)\left(M_{i}+1\right)\left[\frac{L}{B\left(1-p_{i}\right)}+\frac{W_{\min }}{2\left(1-2 p_{i}\right)}-\frac{1}{2\left(1-p_{i}\right)}\right]+\beta \frac{M_{n, i}}{\sum p k_{m, i}}
$$

Nodes use different channels' combination, by sending HELLO packets to the neighbor node to get the information such as the queue length, link bandwidth, the total number of sent and received packets. According to one hop routing metrics WAEED collection, we will set the channel with the minimum one hop routing metric value as the best channel from this node to the next hop node. And establish routing according to the minimum joint routing metrics.

The performance of JCWAEED is compared with the average throughput, average delay and average packet loss rate. The average end-to-end delay and inter-channel interference are used as the metric of routing. The channel with the lowest inter-channel interference is selected as the optimal channel by polling, and the throughput and delay of the network are improved through delay control.

\section{SiMULATION RESULTS}

The simulation parameters are shown in the table below:

TABLE.2 Simulation parameter

\begin{tabular}{|l|l|l|l|}
\hline $\begin{array}{l}\text { Simulation } \\
\text { time }\end{array}$ & $100 \mathrm{~s}$ & $\begin{array}{l}\text { Orthogonal channels } \\
\text { number }\end{array}$ & 3 \\
\hline Simulator & NS2.35 & flow number & 4 \\
\hline $\begin{array}{l}\text { Routing } \\
\text { protocols }\end{array}$ & $\begin{array}{l}\text { MRMC- } \\
\text { AODV/JCEED/JCWAEED }\end{array}$ & $\begin{array}{l}\text { transmission/interference } \\
\text { range }\end{array}$ & $250 \mathrm{~m} / 550 \mathrm{~m}$ \\
\hline Antenna & Omni-directional & channel bandwidth & $11 \mathrm{Mbps}$ \\
\hline $\begin{array}{l}\text { Mesh nodes } \\
\text { number }\end{array}$ & 8 & queue length & $1000 \mathrm{byte}$ \\
\hline $\begin{array}{l}\text { Simulation } \\
\text { area }\end{array}$ & $1000 \mathrm{~m}^{*} 1000 \mathrm{~m}$ & packet loss rate & 0.01 \\
\hline $\begin{array}{l}\text { node radios } \\
\text { number }\end{array}$ & 3 & flow rate & $0.1 \sim 2 \mathrm{Mbps}$ \\
\hline
\end{tabular}

\subsection{Network throughput}

When data flow rate is less than $0.3 \mathrm{Mbps}$, the paper proposed JCWAEED algorithms, JCEED algorithm and the AODV-MRMC algorithm are similar on the average throughput performance. 
When data flow rate is over $0.4 \mathrm{Mbps}$, the average JCWAEED algorithm throughput outperforms JCEED algorithm at the same flow rate. JCWAEED works in high data flow rates because of the improving routing metrics, and it can undertake higher data flow rates.

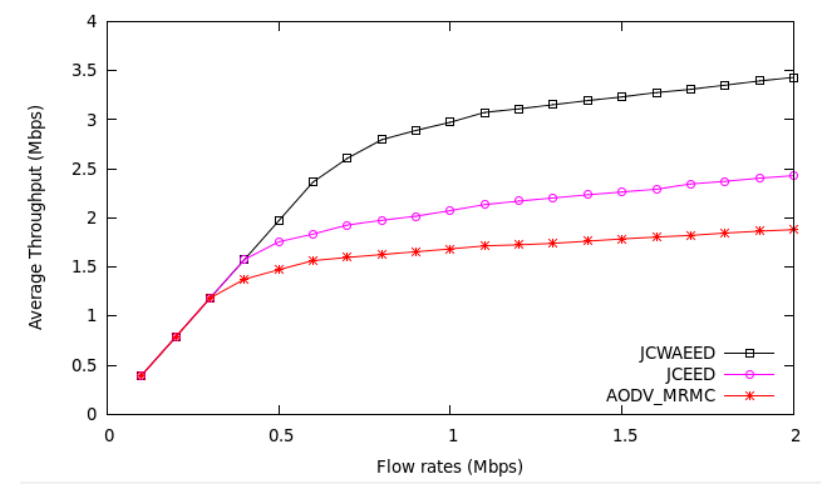

Figure 3. Comparison of average throughput performance in different data flow rates

\subsection{End-to-end delay}

When data flow rate is less than $0.6 \mathrm{Mbps}$, the paper proposed JCWAEED algorithm, JCEED algorithm and the AODV - MRMC algorithm are similar on end-to-end delay performance which is negligible. When data flow rate is around $0.7 \mathrm{Mbps}$, the average end-to-end delay of three algorithms increases rapidly. When data flow rate is over $0.7 \mathrm{Mbps}$, the end-to-end delay of JCWAEED algorithm outperforms JCEED algorithm at the same flow rate. JCWAEED algorithm has the minimum average end-to-end delay.

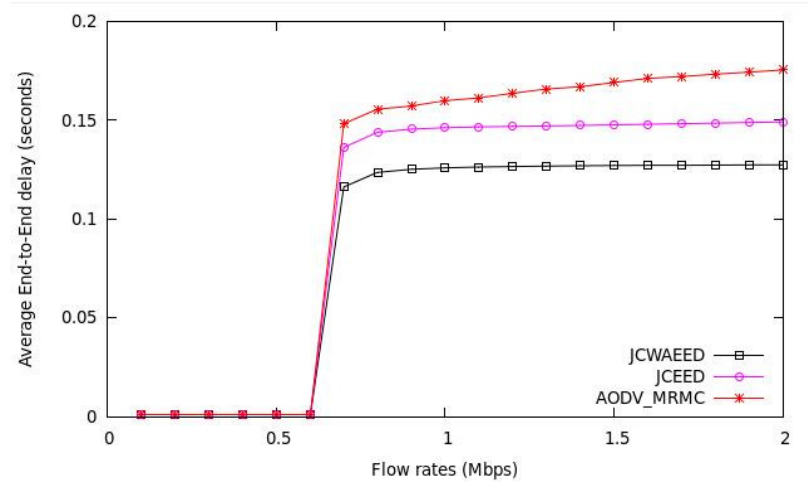

Figure 4. Comparison of end-to-end delay performance in different data flow rates

\subsection{Packet loss}

When data flow rate is less than $0.6 \mathrm{Mbps}$, the paper proposed JCWAEED algorithm, JCEED algorithm and the AODV - MRMC algorithm are similar on packet loss performance, which is negligible. When data flow rate is over $0.6 \mathrm{Mbps}$, the packet loss rate of JCWAEED algorithm outperforms JCEED algorithm at the same flow rate. JCWAEED algorithm has the minimum packet loss. 


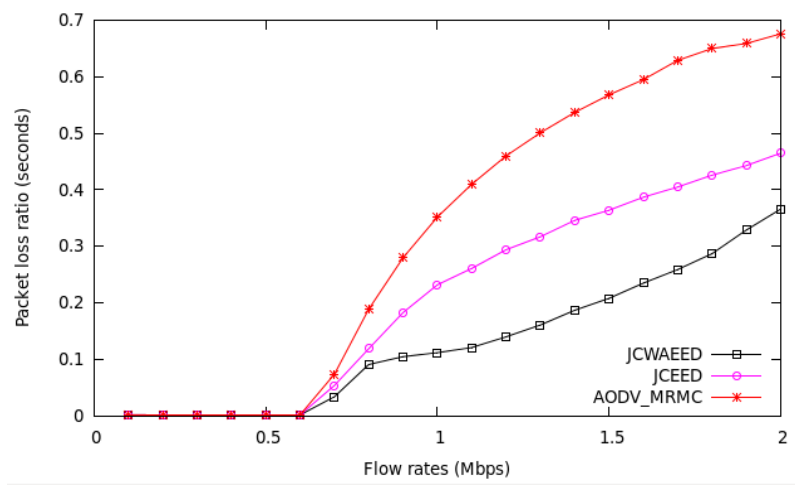

Figure 5. Comparison of packet loss ratio performance in different data flow rates

\section{CONCLUSION}

In this paper, we proposes an effective joint channel assignment and routing optimization protocol called JCWAEED based on the network model and interference model, considering endto-end delay control, interference suppression with factor IF. Additionally, we give the exact calculation formula of transmission delay and queuing delay. Simulations results demonstrate that JCWAEED outperforms other joint design routing protocols in terms of throughput, end-to-end delay and packet loss rate under the influence of different data flow rates.

\section{REFERENCES}

[1] Wang J, Shi W, Jin F. On channel assignment for multicast in multi-radio multi-channel wireless mesh networks: A survey[J].2015, 12(1):122-135.

[2] D. S. De Couto, D. Aguayo, J. Bicket, and R. Morris.A highthroughput path metric for multi-hop wireless routing[J].Wireless Networks, vol. 11, no. 4, pp. 419-434, 2005.

[3] R. Draves, J. Padhye, and B. Zill.Routing in multi-radio, multi-hop wireless mesh networks[C].Conf. on Mobile Computing and Networking, 2004, pp. 114-128.

[4] L. Ma and M. K. Denko, "A routing metric for load-balancing in wireless mesh networks," in Proc. of 21st Int. Conf. on Advanced Information Networking and Applications Workshops, vol. 2, 2007, pp.409-414.

[5] R.F. Ali, A.K. Kiani and A.A. Pirzada.Load Dependent Dynamic Path Selection in Multi-Radio Hybrid Wireless Mesh Networks[C].IEEE Wireless Communications and Networking Conference WCNC, pp. 2044-2049, 2014.

[6] Wu H, Yang F, Tan K. Distributed channel assignment and routing in multiradio multichannel multihop wireless networks Selected Areas in Communications, IEEE Journal on 24(11) 2006 pp. 1972-83.

[7] Pham N T, Hwang W J, Joint Disjoint Path Routing and Channel Assignment in Multi-Radio MultiChannel Wireless Mesh Networks, in IEEE Vehicular Technology Conference, 2008 VTC 2008-Fall IEEE 68th, 2008, pp. 1-5.

[8] Gardellin V, Das S K, Lenzini L, et al. G-PaMeLA: A divide-and-conquer approach for joint channel assignment and routing in multi-radio multi-channel wireless mesh networks[J]. Journal of Parallel \& Distributed Computing, 2011, 71(3):381-396. 
[9] Capone A, Carello G, Filippini I, et al. Routing, scheduling and channel assignment in Wireless Mesh Networks: Optimization models and algorithms[J]. Ad Hoc Networks, 2010, 8(6):545-563.

[10] Sun W, Cong R, Xia F. R-CA: A Routing-Based Dynamic Channel Assignment Algorithm in Wireless Mesh Networks, in IEEE Ubiquitous Intelligence \& Computing and 7th International Conference on Autonomic \& Trusted Computing (UIC/ATC), 2010 7th International Conference on, 2010, pp. 228-32. 